\title{
Diabetes toolkit to link medical and dental care for patients
}

Patients with diabetes should automatically be referred to a dentist as well as other health professionals to check for periodontal disease, according to a new toolkit that is due to be published before the end of the year or early next year.

Some details of the toolkit being prepared by the Chief Dental Officer's (CDO) team in England were revealed at the recent BDIA Dental Showcase 2018 event held in London on 4 to 6 October 2018.

During a speech, Janet Clarke, deputy CDO for England, said the team was trying to align care appropriately between different parts of the health service so patients received the same care regardless of where they lived.

'You will know about the link between diabetes and oral health and that link is becoming better known and the evidence is strong,' said Clarke. 'There is evidence that patients who have diabetes are more likely to have severe periodontal disease but importantly, for patients who are diabetic and have periodontal disease - we can treat that and improve their HbA1c levels - their glycaemic control.

'I think the dental members of the team are aware of that, but possibly, we have some work to do in convincing our medical colleagues of that. That is a piece of work we are doing.

'We are looking to create a toolkit which is very close to being completed that can be used locally with the dental teams working in diabetes to develop common pathways to ensure that a diabetic patient who goes to see their GP is referred to their dentist.'

Currently, such a patient was referred to a podiatrist and an optician but not to a dentist, she explained.

'Similarly, for patients who may go and see their dentist who have periodontal disease, that sets the alarm bells ringing and they are directed along to their GP to start to think about whether there be undiagnosed diabetes. We know there are about two million people who have diabetes but don't know about it.
'We are also developing a commissioning standard so that commissioners are aware of these issues and can start thinking about commissioning dental services that would be helpful for people with diabetes. We have worked closely on this with Diabetes UK and our diabetes colleagues in NHS England.'

Simon O'Neill, Director of Health Intelligence at Diabetes UK, said: 'Periodontitis and Type 2 diabetes share the common risk factor of diet, and therefore there's potential for a combined preventative approach. Recent evidence has also suggested that improved periodontal outcomes could have a direct effect on Type 2 diabetes outcomes, and vice versa.

'We are pleased to be working with our colleagues at NHS England to raise awareness about the connection between periodontitis and Type 2 diabetes. We would support a clear pathway to dental care for people with Type 2 diabetes like what already exists for podiatry, for example.' 